African-American (AA) women may help to explain the disproportionate incidence of HIV/STDs seen in this population. Single women with children by multiple fathers may engage in unprotected sex with the fathers of their children; these men are called Baby Daddies. Baby Daddy sex as a risk factor is complicated and based in a sexual reality grounded in poverty and power. This analysis focused on women who have continued sexual relationships with the father of their children in addition to other sexual relationships. We explored the psychosocial factors surrounding women who engage in unprotected Baby Daddy sex.

Methods We conducted in-depth interviews with AA women living in low-income housing projects in Houston, TX. Study participants were at least 18 years old and involved in multiple sexual relationships. Interviews were conducted in participants' homes and lasted $1 \frac{1}{2} \mathrm{~h}$. Each interview was digitally-recorded and transcribed verbatim. Participants were paid \$20 US for their time. Qualitative analysis was conducted using MAXODA10 software. We identified and organised codes, categories, and themes to form a comprehensive picture of the behaviours of our sample.

Results Our sample included 26 AA women aged 21 to 40 years. Themes that emerged illustrated the contextual environment of women's sexual decisions and included: financial insecurity, parenting, relationship ideals, loneliness, and baby daddy sex. Participants characterised their choice to have unprotected baby daddy sex through the following categories: trust/bond based on shared child, love, compensation for financial support of children, pleasure, and longing for past relationship. These findings identified the importance and implied intimacy of the baby daddy relationships and suggested reasons for unprotected sex.

Conclusions These findings indicate risk implications because they add concurrent sexual relationships with unprotected sex, thus increasing the potential for exposure and transmission of HIV/ STDs. More importantly, this study reflects the lasting importance of a relationship resulting in children and how that relationship influences sexual health. Interventions that focus on poor urban AA women in Houston should acknowledge the cultural constructions of both sexuality and relationships in this population, both related to socioeconomic and power inequalities.

\section{P2-S9.09 HELP ME UNDERSTAND YOUR LAB RESULTS: “AN EXPLORATORY, QUALITATIVE STUDY OF DISCORDANCE BETWEEN BIOLOGIC AND SELF-REPORTED MEASURES OF SEMEN EXPOSURE IN JAMAICA"}

\section{doi:10.1136/sextrans-2011-050108.395}

${ }^{1} \mathrm{~A}$ Bailey, ${ }^{2} \mathrm{M}$ Snead, ${ }^{3} \mathrm{E}$ Costenbader, ${ }^{1} \mathrm{M}$ Townsend, ${ }^{4} \mathrm{C}$ Anderson, ${ }^{2} \mathrm{~L}$ Warner, ${ }^{4} \mathrm{~T}$ Hylton- Kong, ${ }^{3} \mathrm{M}$ Steiner. ${ }^{1}$ University of the West Indies Mona, Kingston, Jamaica; ${ }^{2}$ Centres for Disease Control, Atlanta, USA; ${ }^{3}$ Family Health International, Durham, USA; ${ }^{4}$ Comprehensive Health Centre, Kingston, Jamaica

Background Self-reported survey data on sexual behaviours have limitations which biological measures can complement. We assessed the feasibility of using qualitative interviews to understand discordance between a biological marker of semen exposure and selfreported sexual behaviours among women enrolled in a trial of STI counselling messages. We also investigated reasons women did not follow counselling messages.

Methods Data are from the ACME (Assessing Counselling Message Effectiveness) study. Women age $>18$ years treated for infection at a Jamaica STI clinic were randomly assigned to receive counselling messages to: (1) abstain from sex or (2) abstain but use a condom if they did have sex, during a 6-day treatment period. At follow-up, women reported sexual behaviours in the last 3 days in a quantitative survey and consented to testing for recent semen exposure using a rapid, on-site test for prostate-specific antigen (PSA). Indi- vidual, qualitative interviews were subsequently conducted with a purposive sample of 17 PSA positive women, ten who did not report unprotected sex in the survey (discordant) and seven who did (concordant). Interviewers asked about recent sexual behaviour, sexual partners, counselling messages, and how they may have been exposed to semen. We analysed interview transcripts to assess whether discordance could be explained and to identify reasons counselling messages were not followed.

Results From 8/10 to date, 262 participants completed the ACME trial. Of the 10 qualitative interview participants whose PSA and survey results were discordant, five had reported condom use and five reported abstinence. Interviewers asked these women directly about the inconsistency. This approach variably elicited little concern, discomfort, plausible and implausible explanations from participants. Three condom users and one abstinent participant gave likely reasons for semen exposure, while positive PSA results for two condom users and four abstinent participants remained unexplained. Main reasons given by participants with concordant results for having unprotected sex despite counselling messages were: wanting to please partner, delaying the start of treatment, and not being regular condom users with their partner.

Conclusions Interviewing women about inconsistencies between their self-reported and biologic data are feasible in this context and yielded information for improving future self-reported surveys and understanding the primary study outcomes.

\section{P2-S9.10 HIV TESTING BEHAVIOURS AMONG UNDOCUMENTED CENTRAL AMERICAN IMMIGRANT WOMEN IN HOUSTON, TEXAS, 2010}

doi:10.1136/sextrans-2011-050108.396

J Montealegre, J Risser, B J Selwyn. The University of Texas, School of Public Health, Houston, USA

Introduction Undocumented Central American immigrants in the US are at increased vulnerability to HIV. Almost half of infections are attributed to high-risk heterosexual contact and HIV detection is often delayed. Although HIV testing is a cornerstone of HIV prevention and treatment, we are unaware of any studies that describe HIV testing in this population. The purpose of this paper is to describe HIV testing behaviours among undocumented Central American immigrant women in Houston, Texas.

Methods We recruited 230 Guatemalan, Honduran, and El Salvadoran women, ages $18-50$ years, living in Houston without a US visa or residency papers for an HIV behavioural survey using respondent driven sampling. Present analyses are limited to sexually active participants who provided information on HIV testing $(\mathrm{N}=182)$. Ever testing for HIV was defined as receiving an HIV test at least once during one's lifetime. Prevalence estimates are RDS-adjusted.

Results Sixty seven per cent of women reported ever testing for HIV infection. Among testers, 49\% tested at a public health clinic and $30 \%$ tested at other healthcare facilities; $50 \%$ tested within the past 2 years. The most common reason for getting tested was pregnancy $(50 \%)$ and to get rid of doubt" regarding their partner's infidelity (29\%). Testers were significantly more likely than non-testers to be from Honduras and to have resided in the US for over 5 years. Testers were also older, more educated, and wealthier than nontesters. Testers were more likely than non-testers to have a regular healthcare provider, to have visited a healthcare provider in the past 12 months, and to have healthcare coverage or insurance. Healthcare coverage was predominantly through the indigent healthcare program see Abstract P2-S9.10 Table 1.

Discussion The prevalence of ever testing for HIV among undocumented Central American immigrant women in Houston was high. 